\title{
Drawable and Forbidden Minimum Weight Triangulations *
}

(Extended Abstract)

\author{
William Lenhart ${ }^{1}$, Giuseppe Liotta ${ }^{2}$ \\ 1 Department of Computer Science, Williams College, Williamstown, MA 01267. \\ lonhartecs. villiams.edu \\ 2 Dipartimento di Informatica e Sistemistica, Università di Roma 'La Sapienza', via \\ Salaria 113, I-00198 Roma, Italia. \\ liottaedis. uniromal. it
}

\begin{abstract}
A graph is minimum weight drawable if it admits a straightline drawing that is a minimum weight triangulation of the set of points representing the vertices of the graph. In this paper we consider the problem of characterizing those graphs that are minimum weight drawable. Our contribution is twofold: We show that there exist infinitely many triangulations that are not minimum weight drawable. Furthermore, we present non-trivial classes of triangulations that are minimum weight drawable, along with corresponding linear time (real RAM) algorithms that take as input any graph from one of these classes and produce as output such a drawing. One consequence of our work is the construction of triangulations that are minimum weight drawable but none of which is Delaunay drawable-that is, drawable as a Delaunay triangulation.
\end{abstract}

\section{Introduction and Overview}

Recently much attention has been devoted to the study of combinatorial properties of geometric graphs such as Delaunay triangulations, minimum spanning trees, Gabriel graphs, relative neighborhood graphs, $\beta$-skeleton graphs, and rectangle of influence graphs. This interest has been motivated in part by the importance of these structures in numerous application areas, including computer graphics, pattern recognition, computational morphology, communication networks, numerical analysis, computational biology, and GIS.

Geometric graphs are 2-dimensional straight-line drawings that satisfy some additional geometric constraints. The problem of analyzing the combinatorial properties of a given type of geometric graph naturally raises the question of the characterization of those graphs which admit the given type of straight-line

* Research supported in part by by the EC ESPRIT Long Term Research Project ALCOM-IT under contract 20244. 
drawing. This, in turn, leads to the investigation of the design of efficient algorithms for computing such a drawing when one exists. Although these questions are far from being resolved in general, many partial answers have appeared in the literature. We give two such examples below.

A Gabriel graph (also called Gabriel drawing in the graph drawing literature) is a straight-line drawing such that two vertices $u$ and $v$ are adjacent if and only if the disk having $u$ and $v$ as antipodal points does not contain any other vertex of the drawing. Trees that admit a Gabriel drawing are characterized in [3]. Lubiw and Sleumer [25] show that every maximal outerplanar graph is Gabriel drawable. In [19] the characterization is extended to all outerplanar graphs. The area required by Gabriel drawings is investigated in [24].

Delaunay triangulations are planar straight-line drawings with all internal faces triangles and such that three vertices form a face if and only of the the disk passing through them does not contain any other vertex of the triangulation. To our knowledge, no complete combinatorial characterization of Delaunay drawable triangulations has been given to date. Di Battista and Vismara [7] give a characterization based on a non-linear system of equations involving the angles in the triangulation. Dillencourt has shown that all Delaunay drawable triangulations are 1-tough, and have perfect matchings [11], and that all maximal outerplanar graphs are Delaunay drawable [10]. Dillencourt and Smith [12] show that any triangulation without chords or non facial triangles is Delaunay drawable. A survey on the problem of drawing a graph as a given type of geometric graph can be found in [5].

In this paper we consider a special type of straight-line drawing, a minimum weight drawing, which has applications in areas including computational geometry and numerical analysis. Let $\mathcal{C}$ be a class of graphs, let $P$ be a set of points in the plane, and let $G$ be a graph such that

1. $G$ has vertex set $P$,

2. the edges of $G$ are straight-line segments connecting pairs of points of $P$,

3. $G \in \mathcal{C}$, and

4. the sum of the lengths of the edges of $G$ is minimized over all graphs satisfying $1-3$.

We call such a graph $G$ a minimum weight representative of $\mathcal{C}$. Given a graph $G \in \mathcal{C}$, we say that $G$ has a minimum weight drawing for class $C$ if there exists a set $P$ of points in the plane such that $G$ is a minimum weight representative of $\mathcal{C}$. For example, a minimum spanning tree of a set $P$ of points is a connected, straight-line drawing that has $P$ as vertex set and minimizes the total edge length. So, letting $\mathcal{C}$ be the class of all trees, a tree $G$ has a minimum weight drawing if there exists a set $P$ of points in the plane such that $G$ is isomorphic to a minimum spanning tree of $P$. A minimum weight triangulation of a set $P$ is a triangulation of $P$ having minimum total edge length. Letting $\mathcal{C}$ be the class of all planar triangulations, a planar triangulation $G$ has a minimum weight 
drawing if there exists a set $P$ of points in the plane such that $G$ is isomorphic to a minimum weight triangulation of $P$.

The problem of testing whether a tree admits a minimum weight drawing is essentially solved. Monma and Suri [27] proved that each tree with maximum vertex degree at most five can be drawn as a minimum spanning tree of some set of vertices by providing a linear time (real RAM) algorithm. In the same paper it is shown that no tree having at least one vertex with degree greater than six can be drawn as a minimum spanning tree. As for trees having maximum degree equal to six, Eades and Whitesides [13] showed that it is NP-hard to decide whether such trees can be drawn as minimum spanning trees. The representability of trees as minimum spanning trees in three-dimensional space was studied in [23].

Surprisingly, little seems to be known about the problem of constructing a minimum weight drawing of a planar triangulation. Moreover, it is still not known whether computing a minimum weight triangulation of a set of points in the plane is an NP-complete problem (see Garey and Johnson [14]). Several papers have been published on this last problem, either providing partial solutions, or giving efficient approximation heuristics. See, for example, the work by Meijer and Rappaport [26], Lingas [22], Keil [15], Dickerson et al. [8], Kirkpatrick [16], Aichholzer et al. [1], Cheng and $\mathrm{Xu}$ [4], and Dickerson and Montague [9], Levcopoulos and Krznaric [21, 20].

The problem examined in this paper is that of characterizing those triangulations that admit a minimum weight drawing. In $[18,17]$ it was shown that all maximal outerplanar triangulation are minimum weight drawable and a linear time (real RAM) drawing algorithm for computing a minimum weight drawing of these graphs was given.

Our contributions here are the following:

1. Exploit the relationship between locally minimum weight triangulations and minimum weight triangulations to construct classes of triangulations that are not minimum weight drawable.

2. Exhibit an infinite class of triangulations that can be drawn as minimum weight triangulations but not as Delaunay triangulations.

3. Establish the minimum weight drawability of several non-trivial classes of triangulations, including wheels, spined triangulations, and nested triangulations. (For definitions see Section 2)

4. Investigate the combinatorial structure of minimum weight triangulations by means of the notion of skeleton of a triangulation, that is the graph induced by the set of its internal vertices. We show that any forest can arise as the skeleton of a minimum weight drawable triangulation.

5. Present linear time, real RAM algorithms that accept as input a minimum weight drawable triangulation of one of the types mentioned above and produce as output a minimum weight drawing of that triangulation.

Our algorithmic techniques generalize drawing strategies that have been 
devised in recent years to compute proximity drawings of graphs (see, e.g., $[2,6,19])$.

For reasons of space, the proofs have been omitted in this extended abstract.

\section{Preliminaries}

We first discuss some terminology and define classes of triangulations that are of interest in this paper. We then recall some basic properties of minimum weight triangulations.

Many classes of graphs arise from using some geometric constraint to define edges on a set of points in the plane. Three such classes, Gabriel graphs, Delaunay triangulations and minimum weight triangulations, have already been mentioned. Given a certain class $C$ of graphs defined by such a method, we can define a graph $G$ as being $C$ drawable if $G$ is isomorphic to some member of $C$. Thus in the rest of the paper we will refer to certain triangulations as being, for example, Delaunay drawable, minimum weight drawable, locally minimum weight drawable (see below for a definition) and so forth.

We make particular use of some special types of triangulations. A fon consists of a cycle $a, v_{1}, v_{2}, \ldots, v_{n}$ along with edges from $a$ to each vertex $v_{i}$. The vertex $a$ is called the apex of the fan, and the vertices $v_{i}$ are called the neighbors of $a$. The edges $a v_{i}$ are called the radial edges of the fan.

A wheel graph (or wheel, for brevity) is a triangulation consisting of a cycle and a single vertex $c$, called the center of the wheel, adjacent to all vertices on the cycle. The edges from $c$ to the vertices of the cycle are called the spokes of the wheel.

The class of $k$-nested triangulations can be recursively defined as follows. A three-cycle is a 0 -nested triangulation. For $k>0$, a $k$-nested triangulation is defined to be one having a triangular outer face, the deletion of which results in a $k-1$-nested triangulation. Figure 1 (b) shows a 3-nested triangulation.

Another family of triangulation we will study in this paper is the class of $k$-spined triangulations. Each element of the class is obtained by $k$-spining $K_{4}$. Let $a_{o}, a_{1}, a_{2}$ be the vertices on the outer face and let $c$ be the interior vertex of $K_{4} . k$-spining $K_{4}$ consists of replacing edge $\mathrm{ca}_{2}$ with a path of $k$ vertices such that each new vertex is adjacent to both $a_{0}$ and $a_{1}$. The resulting triangulation is a $k$-spined triangulation. Figure 1 (c) and (d) show a 1-spined and a 2-spined triangulation, respectively. The Delaunay drawability of spined triangulations is studied in [10].

The skeleton of a triangulation is the graph induced by the set of its internal vertices. For example, the skeleton of a maximal outerplanar graph is the empty graph, the skeleton of a wheel graph consists of just one vertex (the center of the wheel). Figure 1 (a) shows a triangulation whose skeleton is a tree. In the figure, the skeleton is highlighted. 


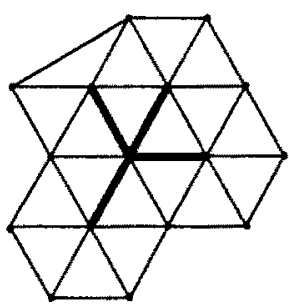

(a)

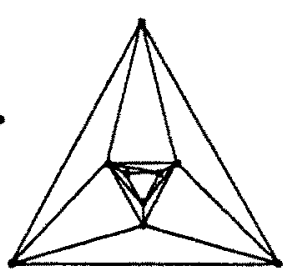

(b)

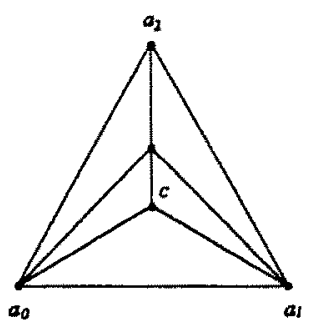

(c)

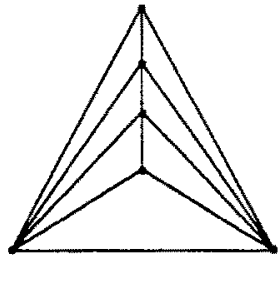

(d)

Fig. 1. (a) A Triangulation whose skeleton is a tree, (b) A 3-nested triangulation, (c) A 1-spined triangulation, and (d) A 2-spined triangulation.

We list some basic properties of minimum weight triangulations.

Property 2.1 A minimum weight triangulation of a set $S$ of points in the plane always includes the shortest edge between two points of $S$ and the all edges of the convex hull of $S$.

Property 2.2 The minimum weight triangulation of a set $S$ of points in the plane need not contain the minimum spanning tree of $S$ as a subgraph.

If $T$ is a triangulation, and abd and bcd are two triangles of $T$ which form a convex quadrilateral, then the operation of replacing edge $b d$ with edge $a c$ is called an edge flip. A triangulation $T$ is locally minimum weight if no single edge flip reduces the weight of $T$.

Property 2.3 If a triangulation cannot be drawn as a locally minimum weight triangulation, then it cannot be drawn as a minimum weight triangulation.

In this paper we will explore the relationship between minimum weight drawable triangulations and Delaunay drawable triangulations. To our knowledge, no complete combinatorial characterization of Delaunay drawable triangulations has been to date given $[10,12,11]$. Di Battista and Vismara [7] give a characterization based on a non linear system of equations involving the angles in the triangulation. Dillencourt $[10,11]$ gives a set of necessary conditions for Delaunay drawability of a triangulation. In the following theorem, $T$ is a triangulation, $P$ a subset of the set $S$ of the vertices of $T,|P|$ is the cardinality of $P$, and $T-P$ is the graph obtained by removing $P$ (and all attached edges) from $T$. 
Theorem 1. [10, 11] If a triangulation $T$ can be drawn as a Delaunay triangulation, then for any given $P \subseteq S$ the following two conditions hold.

1. $T$ is 1-tough, that is, $T-P$ has at most $|P|$ components.

2. $T-P$ contains at most $|P|-2$ components that do not contain any vertex of the outer face of $T$.

\section{Forbidden Triangulations}

In Section 3.1 we show classes of minimum weight forbidden triangulations, i.e. triangulations that are not minimum weight drawable. Interestingly, the triangulations in these classes are also Delaunay forbidden, that is, they cannot be drawn as Delaunay triangulations. This observation leads us to compare minimum weight and Delaunay forbidden triangulations. In Section 3.2 we show an infinite family of triangulations that are minimum weight drawable and Delaunay forbidden.

\subsection{Minimum weight forbidden triangulations}

Our first lemma gives a set of necessary conditions that a triangulation must satisfy in order to be a locally minimum weight triangulation.

To any vertex $v$ and incident face $f$ of a triangulation $T$, we associate a variable $\alpha=\alpha(v, f)$ called the angle of $f$ at $v$; collectively these variables are called the angles of $T$. Any straight-line drawing of $T$ determines values for these variables, and thus certain relations among the angles of $T$ must hold. If the triangulation is to be locally minimum weight, additional relations among the magnitudes of the angles must hold. We define the magnitude of $\alpha$ by $m(\alpha)=1$ if $\alpha$ is obtuse and $m(\alpha)=0$ otherwise.

Lemma 2. Let $T$ be a locally minimum weight triangulation. Then there exists an assignment of values to the angles of $T$ such that the following condition holds:

For each pair of faces $f_{1}$ and $f_{2}$ of $T$ that share an edge e, the two angles $\alpha_{1}$ in $f_{1}$ and $\alpha_{2}$ in $f_{2}$ opposite $e$ satisfy $m\left(\alpha_{1}\right)+m\left(\alpha_{2}\right) \leq 1$.

We can thus use Property 2.3 and Lemma 2 to identify forbidden triangulations.

Theorem 3. Any triangulation containing either the graph of Figure 2 (a) or the graph of Figure $2(b)$ as an induced subgraph is minimum weight forbidden. 


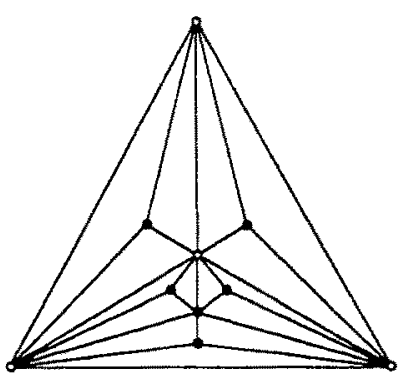

(a)

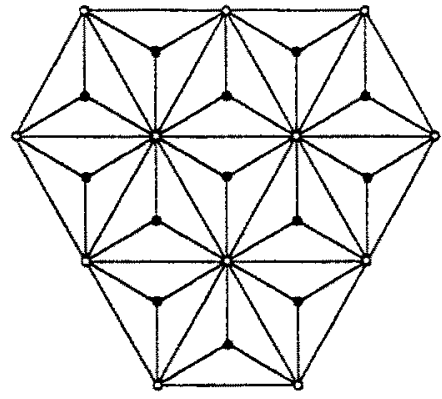

(b)

Fig. 2. Two examples of triangulations that cannot be drawn as minimum weight triangulations.

\subsection{Minimum weight drawable and Delaunay forbidden triangulations}

Lemma 4. Every graph in the class described by Theorem 3 is also Delaunay forbidden.

The above lemma motivates us to investigate the relationship between $\mathrm{De}$ launay forbidden and minimum weight forbidden triangulations.

Lemma 5. The triangulation of Figure 3 is Delaunay forbidden and minimum weight drawable.

The result of Lemma 5 can be generalized to an infinite class of triangulations that are Delaunay forbidden and minimum weight drawable.

Theorem 6. There exists an infinite class of triangulations that are Delaunay forbidden and minimum weight drawable. The graphs of this class are obtained by $k$-spining some $K_{4}$ in the triangulation of Figure 3 (a) for $k>0$.

Figure 3 (c) shows an example of a triangulation that belongs to the class described in Theorem 6, obtained by 1-spining in Figure 3 (a) the $K_{4}$ induced the set of vertices $\left\{a_{0}, c, a_{1}, b\right\}$.

\section{Classes of Minimum Weight Drawable Triangulations}

In this section we construct several classes of minimum weight drawable triangulations. The first two subsections discuss triangulations whose skeleton is either 


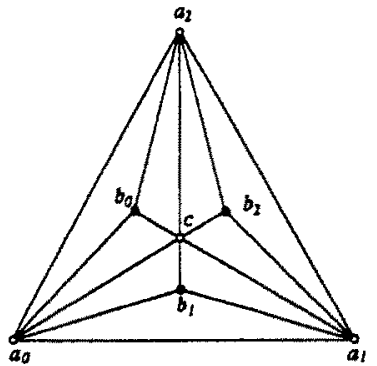

(a)

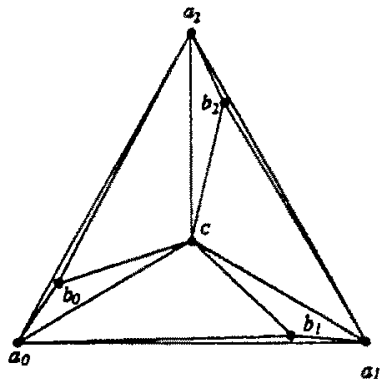

(b)

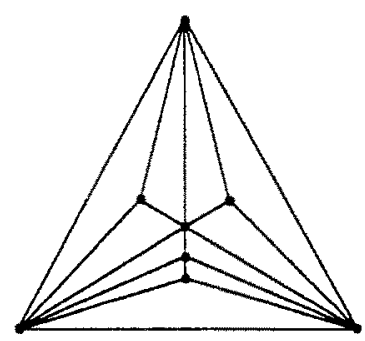

(c)

Fig. 3. (a) A triangulation $T$ that is Delaunay forbidden, (b) A drawing of $T$ that is a minimum weight triangulation, and (c) A forbidden Delaunay and minimum weight drawable triangulation obtained by 1 -spining the subgraph of $T$ induced by the vertices $\left\{a_{0}, c, a_{1}, b\right\}$.

a forest (Subsection 4.1) or a set of nested triangles (Subsection 4.2). Wheel graphs are a special case of the first class of triangulations. The last subsection (Subsection 4.3) is devoted to $k$-spined triangulations.

\subsection{Wheel Graphs and Skeletons}

In the previous section we established that a triangulation $T$ may contain a subgraph which prevents $T$ from being drawn as a minimum weight triangulation. This leads us to examine the structure of the skeleton of the triangulation. We show here that any forest can occur as the skeleton of a minimum weight triangulation. This is accomplished by describing methods for drawing any fan as a minimum weight triangulation, extending a minimum weight drawing of a fan by adding an additional fan, and repeating the previous step to build minimum weight drawings the skeletons of which are arbitrary forests.

The simplest fan of interest to us is a kite: a fan with apex $a$ having exactly three neighbors $b, c, d$. Clearly such a graph is minimum weight drawable; in fact any fan is minimum weight drawable. We will be interested in a certain type of minimum weight drawing of a fan. Let $d(x, y)$ denote the Euclidean distance between points $x$ and $y$ in the plane.

Lemma 7. Let $F=\left\{a, v_{1}, \ldots, v_{n}\right\}$ be a fan with apex $a$, Consider $a$ drawing of $F$ such that $v_{1}, \ldots, v_{n}$ are collinear and for all $1 \leq i<j<k \leq n, d\left(v_{i}, v_{k}\right)>$ $d\left(v_{j}, a\right)$. Then there exists $r>0$ such that if each $v_{i}$ is moved away from a along the line $v_{i} a$ by a distance of at most $r$ so as to form a convex drawing of $F$, the drawing so formed is a minimum weight drawing. 
Using this lemma, a minimum weight drawing of any wheel can be obtained as follows: Delete one of the exterior vertices of the wheel, draw the fan that remains as in the preceding lemma, then replace the deleted vertex suitably far away from the rest of the fan.

We now describe a method for joining a minimum weight drawing of a given triangulation to one of a fan; it will be of use in our later constructions. Let $F$ be a fan with apex $a$ having neighbors $\left\{v_{1}, \ldots, v_{n}\right\}$ which has been drawn according to Lemma 7 . Fix a particular vertex $v_{i}, 1<i<n$ and let $\theta, \tau>0$ be any angles satisfying the following conditions:

1. $\theta+\tau$ is no larger than $\angle v_{i-1} v_{i} v_{i+1}$;

2. if $i \geq 3$ then $\theta+\angle v_{i-2} v_{i-1} v_{i}<\pi$; and

3. if $i \leq n-2$ then $\tau+\angle v_{i+2} v_{i+1} v_{i}<\pi$

where all angles are internal angles of $F$. Let $L_{i-1}$ be the line through $v_{i-1}$ making counterclockwise angle $\theta$ with segment $v_{i-1} v_{i}$, and let $L_{i+1}$ be the line through $v_{i+1}$ making clockwise angle $\tau$ with segment $v_{i+1} v_{i}$. Let $R$ be the quadrilateral region bounded by $L_{i-1}, L_{i+1}, v_{i-1} v_{i}$, and $v_{i+1} v_{i}$, and let $D_{r}\left(v_{1}\right)$ be the circle of radius $r$ centered at $v_{1}$, where $r$ satisfies

$$
r<\frac{1}{2} \min \left\{d\left(v_{i}, v_{k}\right)-d\left(v_{j}, a\right): 1 \leq i<j<k \leq n\right\} .
$$

Finally, $S(r, \theta, \tau)=D_{r}\left(v_{1}\right) \cap R ; S(r, \theta, \tau)$ is called a safe region of $F$ at $v_{i}$.

Lemma 8. Let $F=\left\{a, v_{1}, \ldots, v_{n}\right\}$ be a fan with apex $a$, let $S(r, \theta, \tau)$ be a safe region of $F$ at $v_{i}, 1<i<n$, and let $X$ be a finite subset of $S(r, \theta, \tau)$. Then a minimum weight triangulation of $P=\left\{a, v_{1}, \ldots, v_{n}\right\} \cup X$ can be obtained as the union of a minimum weight drawing of $F$, any minimum weight triangulation of $X \cup\left\{v_{i}\right\}$, and all edges from $v_{i-1}$ and $v_{i+1}$ to $X \cup\left\{v_{i}\right\}$ which cross no edge of the triangulation of $X \cup\left\{v_{i}\right\}$.

Observe that the preceding lemma can be used on a fan consisting of a single kite; this gives a second method for constructing a wheel having $n>3$ spokes: just attach a fan having $n-3$ radial edges to a kite. Note also that given any fan $\bar{F}$, we can choose $X$ in the preceding lemma such that the only minimum weight triangulation of $X \cup\left\{v_{i}\right\}$ is isomorphic to $\bar{F}$. Thus we can view Lemma 8 as providing a method for gluing any fan $\bar{F}$ with apex $u$ to another fan $F$ at (non-apex) vertex $v$ by identifying $u$ with $v$. The ideas contained in the proof of the lemma can also be used to design an efficient algorithm for producing a minimum weight drawing of a wheel. Since constructing a minimum weight drawing of a wheel with three spokes is trivial, it is straightforward to establish the following result.

Theorem 8. Every wheel is minimum weight drawable, and its minimum weight drawing can be computed in linear time in the real RAM model of computation. 
In order to prove that every forest can occur as the skeleton of a minimum weight triangulation, we require a method of attaching several triangulations to an existing fan. The preceding lemma, along with the next definition, will give us one.

Deflnition 10. Let $L$ be a set of line segments in the plane. Two regions $A$ and $B$ of the plane are mutually invisible with respect to $L$ if for each pair of points $a \in A$ and $b \in B$, segment $p q$ intersects some segment of $L$.

Lemma 11. Let $F=\left\{a, v_{1}, \ldots, v_{n}\right\}$ be a fan with apex $a$, let $F^{\prime} \subset F$ be a set of pairwise non-adjacent neighbors of a such that $v_{1}, v_{n} \notin F^{\prime}$, and for each $v_{i} \in F^{\prime}$, consider the kite $\left\{v_{i-1}, v_{i}, v_{i+1}, a\right\}$ in $F$. Choose $r>0$ as in Lemma 8, and, for each $v_{i} \in F^{\prime \prime}$, choose $\left\{\theta_{i}, \tau_{i}\right\}$ so that the safe regions $S\left(r, \theta_{i}, \tau_{i}\right)$ are mutually invisible with respect to $F$. Finally, for each $v_{i} \in F^{\prime}$, let $X_{i}$ be a finite subset of $S\left(r, \theta_{i}, \tau_{i}\right)$. Then a minimum weight triangulation of $P=\left\{a, v_{1}, \ldots, v_{n}\right\} \cup\left\{X_{i}\right.$ : $\left.v_{i} \in F^{\prime}\right\}$ can be obtained as the union of a minimum weight drawing of $F$, any minimum weight triangulations of the sets $X_{i} \cup v_{i}$, for each $v_{i} \in F^{\prime}$, and all edges from each $v_{i-1}$ and $v_{i+1}$ to $X_{i} \cup v_{i}$ which cross no edge of the triangulation of $X_{i} \cup v_{i}$.

Theorem 12. Any forest can be realized as the skeleton of some minimum weight triangulation.

\section{2 $k$-nested Triangulations}

Lemma 13. Let $T$ be a triangulation with triangular outer face, and let $T^{\prime}$ be the triangulation obtained by deleting the vertices on the outer face of $T$. If $T^{\prime}$ has a triangular outer face and is minimum weight drawable, so is $T$.

An application of Lemma 13 is the following.

Theorem 14. Every $k$-nested triangulation is minimum weight drawable, and its minimum weight drawing can be computed in linear time in the real RAM model of computation.

\section{3 k-spined Triangulations}

Lemma 15. Let $T$ be a triangulation with triangular outer face $f_{0}$, and let $T^{\prime}$ be obtained by adding a vertex $v$ in the outer face of $T$ and connecting $v$ with all vertices of $f_{0}$. If $T$ is minimum weight drawable, so is $T^{\prime}$.

An application of Lemma 15 is the following. 
Theorem 16. Every $k$-spined triangulation is minimum weight drawable, and its minimum weight drawing can be computed in linear time in the real RAM model of computation.

\section{Open Problems}

Several problems remain open towards characterizing which graphs admit a minimum weight drawing; among the most relevant are: Does every triangulation whose skeleton is a forest admit a minimum weight drawing? Are there any Delaunay drawable but minimum weight forbidden triangulations? Are there other necessary conditions of the type expressed by Lemma 2 .

\section{References}

1. O. Aichholzer, F. Aurenhammer, S.-W. Chen, N. Katoh, M. Taschwer, G. Rote, and Y.F. Xu. Triangulations intersect nicely. Discrete Comput. Geom., 16:339$359,1996$.

2. P. Bose, G. Di Battista, W. Lenhart, and G. Liotta. Proximity constraints and representable trees. In R. Tamassia and I. G. Tollis, editors, Graph Drawing (Proc. GD '94), volume 894 of Lecture Notes Comput. Sci., pages 340-351. SpringerVerlag, 1995.

3. P. Bose, W. Lenhart, and G. Liotta. Characterizing proximity trees. Algorithmica, 16:83-110, 1996. (special issue on Graph Drawing, edited by G. Di Battista and R. Tamassia).

4. S.-W. Cheng and Y.-F. Xu. Approaching the largest $\beta$-skeleton within a minimum weight triangulation. In Proc. 12th Annu. ACM Sympos. Comput. Geom., pages 196-203, 1996.

5. G. Di Battista, W. Lenhart, and G. Liotta. Proximity drawability: a survey. In R. Tamassia and I. G. Tollis, editors, Graph Drawing (Proc. GD '94), volume 894 of Lecture Notes Comput. Sci., pages 328-339. Springer-Verlag, 1995.

6. G. Di Battista, G. Liotta, and S. H. Whitesides. The strength of weak proximity. In F. J. Brandenburg, editor, Groph Drawing (Proc. GD '95), volume 1027 of Lecture Notes Comput. Sci., pages 178-189. Springer-Verlag, 1996.

7. G. Di Battista and L. Vismara. Angles of planar triangular graphs. SIAM J. Discrete Math., 9(3):349-359, 1996.

8. M. T. Dickerson, S. A. McElfresh, and M. H. Montague. New algorithms and empirical findings on minimum weight triangulation heuristics. In Proc. 11th Annu. ACM Sympos. Comput. Geom., pages 238-247, 1995.

9. M. T. Dickerson and M. H. Montague. A (usually?) connected subgraph of the minimum weight triangulation. In Proc. 12th Annu. ACM Sympos. Comput. Geom., pages 204-213, 1996.

10. M. B. Dillencourt. Realizability of Delaunay triangulations. Inform. Process. Lett., 33:283-287, 1990.

11. M. B. Dillencourt. Toughness and Delaunay triangulations. Discrete Comput. Geom., 5:575-601, 1990. 
12. M. B. Dillencourt and W. D. Smith. Graph-theoretical conditions for inscribability and Delaunay realizability. In Proc. 6th Canad. Conf. Comput. Geom., pages 287292, 1994.

13. P. Eades and S. Whitesides. The realization problem for Euclidean minimum spanning trees is NP-hard. Algorithmica, 16:60-82, 1996. (special issue on Graph Drawing, edited by G. Di Battista and R. Tamassia).

14. M. R. Garey and D. S. Johnson. Computers and Intractability: $A$ Guide to the Theory of NP-Completeness. W. H. Freeman, New York, NY, 1979.

15. M. Keil. Computing a subgraph of the minimum weight triangulation. Comput. Geom. Theory Appl., 4:13-26, 1994.

16. D. G. Kirkpatrick. A note on Delaunay and optimal triangulations. Inform. Process. Lett., 10:127-128, 1980.

17. W. Lenhart and G. Liotta. Drawing outerplanar minimum weight triangulations. Inform. Process. Lett., 6(12):253-260, 1996.

18. W. Lenhart and G. Liotta. How to draw outerplanar minimum weight triangulations. In F. J. Brandenburg, editor, Graph Drawing (Proc. GD '95), volume 1027 of Lecture Notes Comput. Sci., pages 373-384. Springer-Verlag, 1996.

19. W. Lenhart and G. Liotta. Proximity drawings of outerplanar graphs. In S. North, editor, Graph Draving (Proc. GD '96), volume 1190 of Lecture Notes Comput. Sci., pages 286-302. Springer-Verlag, 1997.

20. C. Levcopoulos and D. Krznaric. Quasi-greedy triangulations approximating the minimum weight triangulation. In Proc. 7th ACM-SIAM Sympos. Discrete Algorithms, pages 392-401, 1996.

21. C. Levcopoulos and D. Krznaric. Tight lower bounds for minimum weight triangulation heuristics. Information Processing Letters, (57):129-135, 1996.

22. A. Lingas. A new heuristic for minimum weight triangulation. SIAM J. Algebraic Discrete Methods, 8(4):646-658, 1987.

23. G. Liotta and G. Di Battista. Computing proximity drawings of trees in the 3dimensional space. In Proc. 4th Workshop Algorithms Data Struct., volume 955 of Lecture Notes Comput. Sci., pages 239-250. Springer-Verlag, 1995.

24. G. Liotta, R. Tamassia, I. G. Tollis, and P. Vocca. Area requirement of Gabriel drawings. In Algorithms and Complexity (Proc. CIAC' 97), volume 955 of Lecture Notes Comput. Sci., pages 239-250. Springer-Verlag, 1995.

25. A. Lubiw and N. Sleumer. Maximal outerplanar graphs are relative neighborhood graphs. In Proc. 5th Canad. Conf. Comput. Geom., pages 198-203, 1993.

26. H. Meijer and D. Rappaport. Computing the minimum weight triangulation of a set of linearly ordered points. Information Processing Letters, (42):35-38, 1992.

27. C. Monma and S. Suri. Transitions in geometric minimum spanning trees. Discrete Comput. Geom., 8:265-293, 1992. 